\title{
Antimicrobial and antioxidant activities of extracts from Lycoris aurea bulbs, an Amaryllidaceae plant
}

\author{
Qiaomei Ru *, Guoping Ren ${ }^{a}$ and Xuebing Zhang ${ }^{\text {b }}$ \\ Hangzhou Wanxiang Polytechnic, Department of Health and Agriculture, Hangzhou 310023, China. \\ * Corresponding author email: 1483091098@qq.com \\ a392561314@qq.com,b369317246@qq.com
}

Keywords: Lycoris aurea; Flavonoids; Antimicrobial activity; Antioxidant activity.

\begin{abstract}
This study was aim to explore the antimicrobial and antioxidant activities of flavonoid and polysaccharide extracts from bulbs of Lycoris aurea. The disc diffusion method was used to evaluate the antimicrobial activity of flavonoid and polysaccharide extracts from the bulbs of L. aurea. The antioxidant activity was determined by scavenging of hydroxyl and 1,1-diphenyl-2-picrylhydrazine (DPPH) free radicals, and reducing ability. Flavonoids were effective on 7 microorganisms, but polysaccharides had no effect. Flavonoids had better hydroxyl free radical scavenging activity and reducing capacity than polysaccharides, but weaker than ascorbic acid. However, flavonoids had similar DPPH free radical activity to ascorbic acid. The strong antibacterial and antioxidant activities of flavonoids were mainly attributed to the high content of total phenols in the extract, which was $76.71 \pm 4.42 \mathrm{mg} / \mathrm{g}$. The study shows that flavonoids from bulbs of Lycoris aurea could be regarded as a new potential source of natural bacterial inhibitors and antioxidants.
\end{abstract}

\section{Introduction}

The production of reactive oxygen species is a common reaction and an essential biological process during normal cell metabolism. However, excess reactive oxygen species has been linked with numerous chronic health problems such as cancers, inflammation, aging, and atherosclerosis. On the other hand, pathogenic bacteria can cause food-borne illness and a wide variety of infectious diseases. A number of studies have shown that medicinal plants exhibit antimicrobial and antioxidant activities [1].

Plants of the Amaryllidaceae family are well known not only for their ornamental value but also for the alkaloids they produce, some of which have been found to exhibit interesting pharmacological and/or biological properties [2].The most studied of those alkaloids is galanthamine,which is a long-acting, selective, reversible, and competitive inhibitor of acetylcholinesterase; therefore, it is a reasonable approximation of the ideal concept of symptomatic Alzheimer's disease therapy. The yield of galanthamine in Leucojuma estivum varies from 1.00 to $20.00 \mathrm{mg} / \mathrm{g}$ dry weight (DW), and a much higher galanthamine content of $74.00 \mathrm{mg} / \mathrm{g}$ DW has been found in a sample of Phaedranassa negistrophylla from Peru. Lycoris aurea (L. aurea), a member of the Amaryllidaceae family, is widely distributed in the tropical and warm temperature regions of China. It has been used from ancient times in floriculture and medicine. It was found that galanthamine contents in the bulbs of L. aurea, depending on the season, ranged from 0.26 to $0.57 \mathrm{mg} / \mathrm{g}$ DW. Although L. Aurea contains much lower amounts of galanthamine than Phaedranassa negistrophylla, it has the highest galanthamine content among plants we can found in China[3]. Polysaccharides, another important component, are also present in large amounts in the Amaryllidaceace family, especially in the bulb. Our laboratory found that the bulb of L. aurea contained, depending on the seasons, from $10.87 \%$ to $37.44 \%$ polysaccharides (per fresh weight)[4]. However, to the best of our knowledge, there are no published reports related on the bioactivities activities of alkaloids and polysaccharides from L. aurea bulbs. 
Accordingly, the mechanism of action of this plant has been partly related to its antimicrobial and antioxidant activities. The present study was aimed to investigate the antimicrobial and antioxidant effects of the flavonoids and polysaccharides from L. aurea bulbs.

\section{Materials and Methods}

\subsection{Plant Material and Chemicals}

The bulbs of L. aurea were collected from Chengdu, Sichuan, China, and identified by Herbarium Specimen Search of Xiamen University. The voucher specimens were deposited in the School of Life Sciences, Xiamen University. Folin-Ciocalteu reagent, ascorbic acid and DPPH were purchased from Sigma Chemical Co. (St. Louis, MO, USA). Other chemicals and solvents used were of analytical grade.

\subsection{Isolation of Polysaccharides and Flavonoids from L. aurea Bulbs}

\subsubsection{Isolation of Flavonoids}

$50 \mathrm{~g}$ dried L. aurea bulbs powder was placed in a reflux apparatus. Extraction was performed with $500 \mathrm{~mL}$ of $85 \%(\mathrm{v} / \mathrm{v})$ aqueous ethanol for $60 \mathrm{~min}$ at $80^{\circ} \mathrm{C}$. The crude extract was filtered. The solution was concentrated under reduced pressure. The crude flavonoids-enriched extract was purified using a column $(25 \times 1.5 \mathrm{~cm} 2)$ packed with AB-8 macroporous adsorption resin. The conditions for purifying the flavonoids by $\mathrm{AB}-8$ resin were: injecting concentration $3.75 \mathrm{mg} / \mathrm{mL}, \mathrm{pH}=5$, injecting velocity $2.0 \mathrm{~mL} / \mathrm{min}, 40 \%(\mathrm{v} / \mathrm{v})$ ethanol as desorption solvent, desorption velocity of flow $1.5 \mathrm{~mL} / \mathrm{min}$. The purified extract of flavonoids was collected and evaporated at $50^{\circ} \mathrm{C}$, and was then freeze-dried for determination of flavonoid content and antioxidant property.

\subsubsection{Isolation of Polysaccharides from L. aurea Bulbs}

The polysaccharides of L. aurea bulbs was isolated according to the method described by Ru et al.[5] with a minor modification. Lycoris aurea bulbs powder (50 g each) was extracted with 20 volumes of distilled water at $100^{\circ} \mathrm{C}$ for $2 \mathrm{~h}$. The suspension was centrifuged $(8000 \times \mathrm{g})$ for $15 \mathrm{~min}$, and the supernatant was concentrated under vacuum. The protein in the product of condensation was deproteinized using the Sevag reagent. After removal of the Sevag reagent, 4 volumes of anhydrous ethanol were added to this concentrate, and the mixture was stirred at room temperature for $20 \mathrm{~min}$ and then left at $4^{\circ} \mathrm{C}$ overnight. The precipitate was collected by centrifugation $(8000 \times \mathrm{g})$ for $15 \mathrm{~min}$, subsequently washed three times with anhydrous ethanol, acetone and ether, respectively, then dissolved in distilled water and dialyzed in dialysis bag (molecular weight cut-off, $12 \mathrm{kDa}$ ) against distilled water at room temperature for three successive days. The retained fraction was recovered, concentrated under vacuum and lyophilized to obtain crude polysaccharide. Crude polysaccharide was dissolved in distilled water, centrifuged, and then the supernatant was applied to a column of anion exchange chromatography on DEAE-Sepharose Fast Flow column $(100 \times 2.6 \mathrm{~cm} 2)$, eluting with $0-2 \mathrm{M}$ gradient $\mathrm{NaCl}$ solution. The fractions eluted with $0-2 \mathrm{M}$ gradient $\mathrm{NaCl}$ were collected, concentrated, dialyzed and lyophilized to get purified polysaccharides.

\subsection{Content Determination of Flavonoids, Polysaccharides and Total Phenol}

The content of flavonoids was determined using a colormetric method. Total phenolic content was measured by using the Folin-Ciocalteu reagent and using gallic acid as standard. The content of polysaccharides was determined using the phenol-sulphuric acid method.

\subsection{Antimicrobial Activity}

\subsubsection{Microbial Strains}

Eight microbial strains were used to assess the antimicrobial properties of the extracts, including the Gram-positive bacteria S. aureus subsp. Aureus (1.128), B. subtilis (1.1630) and B. cereus (1.126), as well as the Gram-negative bacteria E. coli (1.1369), and S. typhimurium (1.1194), and 3 fungi M. 
racemosus (3.3446), A. oryzae (3.5232) and R. stolonifer (3.4110). Microorganisms were provided by the Institute of Microbiology Chinese Academy of Sciences.

All microorganisms were maintained at $-20^{\circ} \mathrm{C}$ under appropriate conditions and regenerated twice before use. Microorganisms were cultured aerobically at $37^{\circ} \mathrm{C}$ in nutrient agar (NA) medium for bacteria, and at $30^{\circ} \mathrm{C}$ in potato dextrose agar (PDA) medium for fungi.

\subsubsection{Inhibitory Effect by the Disc Diffusion Method}

Antimicrobial activity assays of the extracts were carried out by the disc diffusion method. A suspension of the tested microorganism $(\approx 108 \mathrm{CFU} / \mathrm{mL})$ was spread on fifteen millilitres of NA or PDA. The wells were made, and the extracts $(20 \mathrm{mg} / \mathrm{mL})$ were added to wells $(20 \mu \mathrm{L})$ and the same volume $(20 \mu \mathrm{L})$ of ethanol and water were used as negative controls. Under the same conditions, ampicillin $(50 \mu \mathrm{g} / \mathrm{disk})$ and nystatin $(50 \mu \mathrm{g} / \mathrm{disk})$ were used as positive controls. The mediums were incubated at $37^{\circ} \mathrm{C}$ for $24 \mathrm{~h}$ (bacteria) or at $30^{\circ} \mathrm{C}$ for $48 \mathrm{~h}$ (fungi). At the end of incubation, inhibition zones were measured (mm).

\subsubsection{Determination of Minimum Inhibitory Concentration (MIC)}

The MIC was determined by the micro-dilution method. In brief, 96-well plates were prepared by dispensing $100 \mu \mathrm{L}$ of the inoculum into each well. Aliquots $(100 \mu \mathrm{L})$ of the extract stock solutions were added into the first wells. The inoculum and extract in first wells were mixed thoroughly before transferring $100 \mu \mathrm{L}$ of the resultant mixtures to second wells. Then, the same procedure was repeated for inoculum mixtures in six consecutive wells, thus creating a serial dilution of the test materials. The plates were cultured at $37^{\circ} \mathrm{C}$ for $24 \mathrm{~h}$ (bacteria) or at $30^{\circ} \mathrm{C}$ for $48 \mathrm{~h}$ (fungi) to observe if there was any bacterial or fungal growth. The MIC was assumed as the lowest concentration of extracts that inhibited growth. Each assay in this experiment was repeated thrice.

\subsection{Antioxidant Property}

The hydroxyl radicalscavenging activity was assessed by the method of Smirnoff and Cumbes[6]. According to the method of Braca et al.[7], the measurement of DPPH radical scavenging ability was carried out. The reducing power was assessed according to the method of Dorman and Hiltunen[8].

\subsection{Statistical Analysis}

Each experiment was performed in triplicate and the result was expressed as mean \pm standard deviation of three replications. p vaule $<0.05$ was regarded as significant.

\section{Results and Discussion}

\subsection{Flavonoids and Polysaccharides from L. aurea Bulbs}

Flavonoids and polysaccharides are widely found in many plants, and they are the active ingredients of some Chinese herbal medicines and play an important role in a variety of pharmacological activities, such as removing free radicals, anticancer effects, and diastolic blood vessels. Hence, the determination ofthe flavonoids and polysaccharides contentin L. aurea bulbs is of great significance. The yields of flavonoids and polysaccharides from L. aurea bulbs on a dry weight basis were $16.13 \pm 1.38 \mathrm{mg} / \mathrm{g}$ and $64.82 \pm 2.42 \mathrm{mg} / \mathrm{g}$, respectively. For the polysaccharidic fraction, a level of $43.78 \pm 2.96 \mathrm{mg} / \mathrm{g}$ was shown for the neutral polysaccharides, whereas $3.04 \pm 0.21 \mathrm{mg} / \mathrm{g}$ was found for the acidic polysaccharides. The total phenolic contents of the flavonoid and polysaccharidic fractions were investigated, which were $76.71 \pm 4.42$ and $0.69 \pm 0.04 \mathrm{mg} \mathrm{GAE} / \mathrm{g}$, respectively. It was confirmed that some polysaccharides are linked to phenols, and might be co-extracted using polar solvents[9]. The results indicated that flavonoids had a higher total phenolic content than polysaccharides $(\mathrm{p}<0.05)$, in agreement with the report of Ru et al.[5] that the polysaccharides had a lower total phenolic content than the flavonoids of tobacco leaves. 


\subsection{Antimicrobial Activities of Flavonoids and Polysaccharides from L. aurea Bulbs}

The antibacterial activities of $\mathrm{L}$. aurea bulb extracts against foodborne and pathogenic microorganisms were evaluated (Table 1). The inhibition zones of the extracts for each assay on the tested microorganisms showed a reverse relationship with the MIC. At a low concentration, a large inhibition zone indicated strong antimicrobial activity. These results indicated that flavonoids were active against all tested microorganisms, whereas polysaccharides did not show any activity. The antimicrobial effect increased with increased amount of flavonoids, with the MIC ranging from 1.25 $\mathrm{mg} / \mathrm{mL}$ to $2.5 \mathrm{mg} / \mathrm{mL}$ for bacteria and $5.0 \mathrm{mg} / \mathrm{mL}$ for fungi. The antimicrobial activities of the tested extracts were compared with those of the standard antibiotics, ampicillin (for bacteria), and nystatin (for fungi). Results showed that the standard antibiotics had stronger activities than the extracts. The negative controls (ethanol and water) did not show any inhibitory effect on the tested organisms. Among the extracts of Thymus cappadocicus Boiss, the ethanolic extract is the most effective against the microorganisms tested, whereas the water extract does not exhibit any activity[10]. Serrano et al.[11] also reported that the aqueous extract of Satureja montana does not have antibacterial activity, whereas the ethanolic extract is more effective in inhibiting bacterial growth.

Natural products may be a particularly rich source of antimicrobial agents. We proposed, for the first time, the use of L. aurea bulbs as a source of antimicrobials. Our antimicrobial assays, including the disc diffusion method and MIC measurement, indicated that S. aureus was the most susceptible microorganism, showing the largest inhibition zone and lowest MIC value, respectively. A study that screened for antibacterial activity in 191 plant extracts belonging to 30 families of plants from Sabah, Malaysia shows similar results[12]. This result consistency is important because S. aureus is well known for being resistant to a number of phytochemicals and for the production of several types of enterotoxins that cause gastroenteritis[13]. Therefore, L. aurea bulbs may be a potential natural antimicrobial agent in food systems that can prevent the growth of food-borne bacteria and thereby extend the shelf life of processed foods.

TABLE 1. Antibacterial activity of the extracts from Lycoris aurea bulbs.

\begin{tabular}{ccccccccc}
\hline & \multicolumn{2}{c}{$\begin{array}{c}\text { Polysaccharides } \\
(20 \mathrm{mg} / \mathrm{mL})\end{array}$} & \multicolumn{2}{c}{$\begin{array}{c}\text { Flavonoids } \\
(20 \mathrm{mg} / \mathrm{mL})\end{array}$} & Ampicillin & Nystatin & Ethanol & Water \\
\cline { 2 - 9 } Microorganisms & $\begin{array}{c}\mathrm{IZ} \\
(\mathrm{mm})\end{array}$ & $\begin{array}{c}\mathrm{MIC} \\
(\mathrm{mg} / \mathrm{mL})\end{array}$ & $\begin{array}{c}\mathrm{IZ} \\
(\mathrm{mm})\end{array}$ & $\begin{array}{c}\mathrm{MIC} \\
(\mathrm{mg} / \mathrm{mL})\end{array}$ & $\begin{array}{c}\mathrm{IZ} \\
(\mathrm{mm})\end{array}$ & $\begin{array}{c}\mathrm{IZ} \\
(\mathrm{mm})\end{array}$ & $\begin{array}{c}\mathrm{IZ} \\
(\mathrm{mm})\end{array}$ & $\begin{array}{c}\mathrm{IZ} \\
(\mathrm{mm})\end{array}$ \\
\hline $\begin{array}{c}\text { Gram-positive } \\
\text { bacteria }\end{array}$ & & & & & & & & \\
S. aureus & $6.4 \pm 0.1$ & $>20$ & $25.0 \pm 0.5$ & 1.25 & $40.7 \pm 1.2$ & $\mathrm{ND}$ & $6.0 \pm 0.1$ & $6.0 \pm 0.1$ \\
B. subtilis & $6.2 \pm 0.2$ & $>20$ & $19.8 \pm 0.3$ & 2.5 & $21.8 \pm 1.1$ & $\mathrm{ND}$ & $6.1 \pm 0.2$ & $6.0 \pm 0.2$ \\
B. cereus & $6.3 \pm 0.1$ & $>20$ & $18.1 \pm 0.8$ & 2.5 & $24.2 \pm 0.8$ & ND & $6.0 \pm 0.2$ & $6.0 \pm 0.1$ \\
Gram-negative & & & & & & & & \\
bacteria & & & & & & & & \\
E. coli & $6.6 \pm 0.6$ & $>20$ & $13.2 \pm 0.2$ & 2.5 & $23.2 \pm 0.9$ & $\mathrm{ND}$ & $6.2 \pm 0.3$ & $6.1 \pm 0.1$ \\
S. typhimurium & $6.2 \pm 0.3$ & $>20$ & $16.2 \pm 0.4$ & 2.5 & $26.4 \pm 1.4$ & ND & $6.1 \pm 0.2$ & $6.0 \pm 0.2$ \\
$\quad$ Fungi & & & & & & & & \\
M. racemosus & $6.5 \pm 0.4$ & $>20$ & $11.5 \pm 0.5$ & 5.0 & ND & $32.2 \pm 1.1$ & $6.2 \pm 0.3$ & $6.2 \pm 0.2$ \\
A. oryzae & $6.6 \pm 0.3$ & $>20$ & $12.2 \pm 0.4$ & 5.0 & ND & $24.6 \pm 0.8$ & $6.4 \pm 0.5$ & $6.3 \pm 0.6$ \\
R. stolonifer & $6.4 \pm 0.7$ & $>20$ & $9.9 \pm 0.6$ & 5.0 & ND & $26.1 \pm 0.9$ & $6.1 \pm 0.2$ & $6.1 \pm 0.1$ \\
\hline
\end{tabular}

\subsection{Antioxidant Activities of Flavonoids and Polysaccharides from L. aurea Bulbs}

Considering the multifaceted aspects of antioxidants and their reactivities, several antioxidant assays were conducted. The antioxidant activities of L. aurea bulb extracts were evaluated using the biochemical methods of hydroxyl and DPPH radical scavenging analyses, as well as reducing power assay. 


\subsubsection{Scavenging Ability on Hydroxyl Radicals}

Hydroxyl radicals are extremely reactive free radicals formed in biological systems, and are considered highly damaging species in free-radical pathology. These radicals are capable of damaging almost every molecule found in living cells. Therefore, assessing the protective ability of the L. aurea bulb extracts against hydroxyl radicals is important. The scavenging abilities of the extracts for hydroxyl radicals were shown in Fig. 1. The hydroxyl radical scavenging abilities of flavonoids and polysaccharides were concentration dependent. These results proved that flavonoids were a good scavenger for hydroxyl radicals. The antioxidants minimized the concentration of Fe2+ in the Fenton reaction, and the scavenging abilities of the antioxidants may be due to the active hydrogen donating ability of hydroxyl substitutions of antioxidants. For flavonoids, the keto group at C-4' and hydroxy group at C-3' or C-5' of the C-ring were effective in scavenging superoxide anion, and the greater the number of hydroxy groups, the better is the scavenging activity[14]. However, the scavenging abilities of the two extracts on hydroxyl radicals were both relatively lower than that of ascorbic acid. A similar phenomenon was also observed in L. barbarum L., which is also a traditional Chinese herb[15] and tobacco leaves[5].

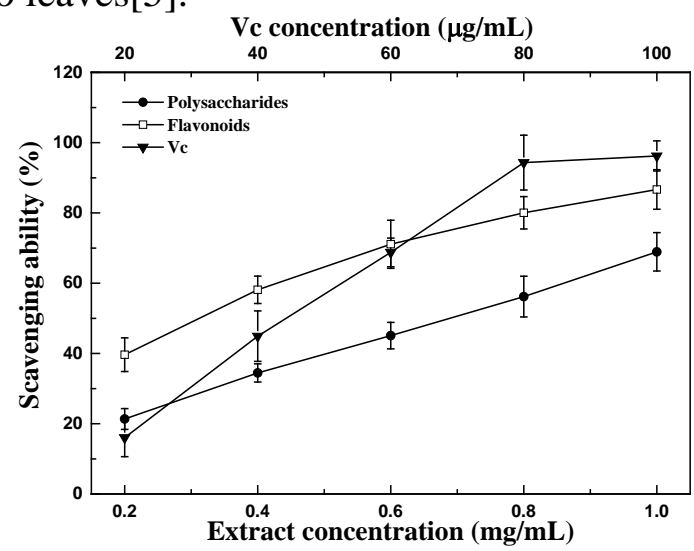

Figure 1. Scavenging activities of the extracts from L. aurea bulbs on hydroxyl radicals.

\subsubsection{Scavenging Ability on DPPH Radicals}

The model of scavenging DPPH radicals is widely accepted as a tool to evaluate the free-radical scavenging activities of materials. Fig. 2 suggested that the scavenging abilities of flavonoids and polysaccharides on DPPH radicals were concentration dependent. The flavonoids had a higher scavenging ability for DPPH radicals than polysaccharides. This finding can be attributed to the higher potential of flavonoids for reacting with DPPH radicals and converting them to more stable products, thereby terminating radical chain reactions. The scavenging abilities for DPPH radicals of the flavonoids and polysaccharides were lower than that of ascorbic acid. In a previous study, the water extract of mulberry leaves was found to be weaker in DPPH radical scavenging than the methanolic extract[16].

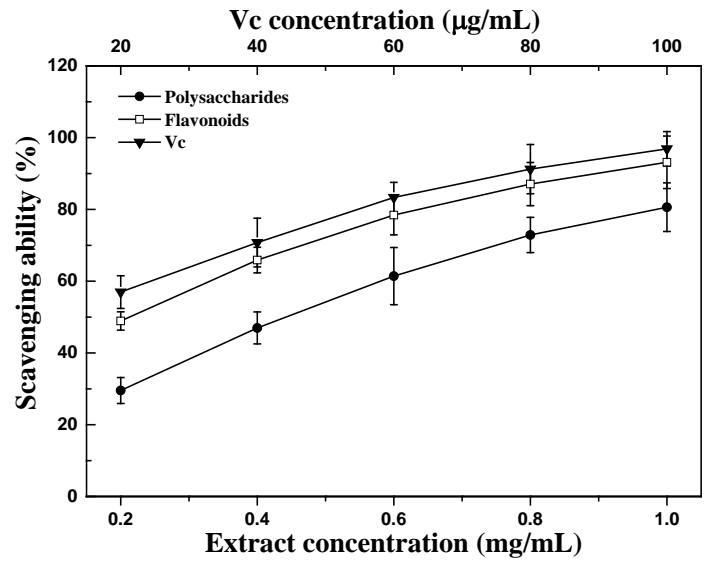

Figure 2. Scavenging activities of the extracts from L. aurea bulbs on DPPH radicals. 


\subsubsection{Reducing PowerAbility}

A good correlation between antioxidant activity and reducing power in some plant extracts has been established. Therefore, reducing the capacity of a compound may serve as a significant indicator of its potential antioxidant activity. Accordingly, the effect of the L. aurea bulbs extracts on the reducing power was determined, and the results were shown in Fig. 3. In this assay, the reducing power was found to be concentration dependent for the flavonoids and polysaccharides. The flavonoids showed an obviously higher ability than the polysaccharides $(p<0.05)$, but a lower one than ascorbic acid. The high reducing power of the flavonoids suggested its remarkable potential to donate electrons to reactive free radicals, convert them into more stable non-reactive species, and then terminate the free-radical chain reaction[17]. Similarly, polysaccharides had the weakest reducing power activity than flavonoids of Lycium barbarumL[15] and tobacco leaves[5].

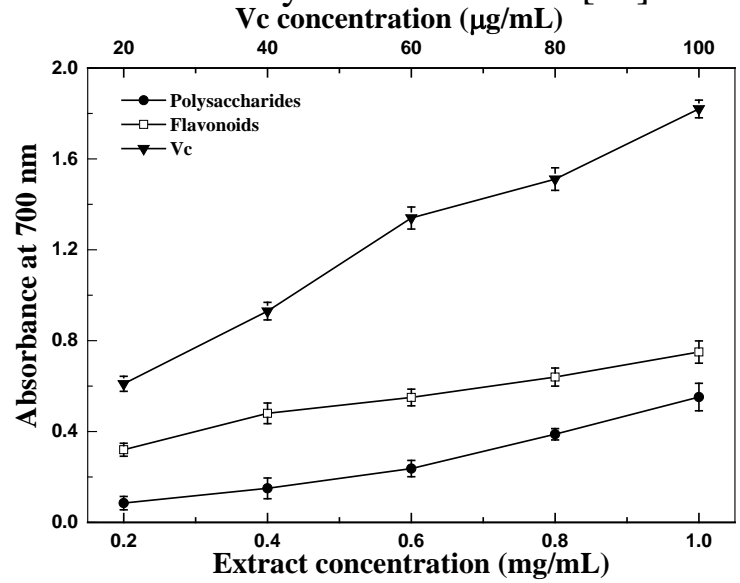

Figure 3. Reducing power of the extracts from L. aurea bulbs.

\subsubsection{EC E0 $_{50}$ Values for Antioxidant Properties}

Concentration of sample at which the inhibition percentage reaches $50 \%$ is the $\mathrm{IC}_{50}$ value. The $\mathrm{IC}_{50}$ was utilized to compare the radical scavenging activity from different extracts. The $\mathrm{EC}_{50}$ values of flavonoids and polysaccharides for free radical scavenging activities, as well as reducing power abilities, were summarized in Table 2. $\mathrm{EC}_{50}$ values of flavonoids for scavenging activities on hydroxyl and DPPH radicals were 0.32 and $0.22 \mathrm{mg} / \mathrm{mL}$, respectively, which were lower than those of polysaccharides $\left(0.71\right.$ and $0.46 \mathrm{mg} / \mathrm{mL}$, respectively). The $\mathrm{EC}_{50}$ value of flavonoids $(0.48 \mathrm{mg} / \mathrm{ml})$ for reducing power was significantly higher than that of polysaccharides $(0.96 \mathrm{mg} / \mathrm{mL})$. Our results agreed with the finding of Wang et al.[15] who found that the antioxidant activity of L. barbarum extracts was mainly attributed to flavonoids.

$\mathrm{EC}_{50}$ value is negatively related to the antioxidant activity, as it expresses the amount of antioxidant needed to decrease the radical concentration by $50 \%$. The lower the $\mathrm{EC}_{50}$ value, the higher is the antioxidant activity of the tested sample. Sun et al.[18] have reported the antioxidant properties of flavonoids from persimmon (Diospyros kaki L.) leaves, a traditional Chinese medicine, and indicated that the $\mathrm{EC}_{50}$ values for hydroxyl and DPPH radical-scavenging ability were 70.64 $\mu \mathrm{g} / \mathrm{mL}$ and $96.36 \mu \mathrm{g} / \mathrm{mL}$, respectively, which were much lower than that of flavonoids from L. aurea bulbs. However, the $\mathrm{EC}_{50}$ values of flavonoids obtained by ethanol extract method from sweet potato (Ipomoea batatas L.) leaves, one of the most important economic crops, for hydroxyl radical-scavenging ability were about $0.8 \mathrm{mg} / \mathrm{mL}$ [19], indicating much weaker antioxidant ability than the flavonoids in this experiment. In addition, with regard to reducing power, the flavonoids showed higher ability than phenolic and polysaccharidic extracts of a popular medicinal mushroom, G. lucidum fruiting body, which were $0.62 \mathrm{mg} / \mathrm{mL}$ and $0.81 \mathrm{mg} / \mathrm{mL}$, respectively [20]. Therefore, it was indicate that flavonoids from $\mathrm{L}$. aurea bulbs are effective in scavenging free radicals and have the potential to be a powerful antioxidant. 
TABLE 2. $\mathrm{EC}_{50}$ values of the extracts of L. aurea bulbs.

\begin{tabular}{cccc}
\hline & \multicolumn{3}{c}{ EC50 value $(\mathrm{mg} / \mathrm{mL})$} \\
\cline { 2 - 4 } Samples & Hydroxyl radicals & DPPH radicals & Reducing power \\
\hline Polysaccharides & $0.71 \pm 0.016 \mathrm{a}$ & $0.46 \pm 0.012 \mathrm{a}$ & $0.96 \pm 0.024 \mathrm{a}$ \\
Flavonoids & $0.32 \pm 0.009 \mathrm{~b}$ & $0.22 \pm 0.007 \mathrm{~b}$ & $0.48 \pm 0.017 \mathrm{~b}$ \\
Ascorbic acid & $0.043 \pm 0.0006$ & $<0.02$ & $<0.02$ \\
\hline
\end{tabular}

Different letters in the same column indicate statistical significance $(\mathrm{p}<0.05)$.

\subsection{Relation between Bioactivities and Phenolic Content}

The phenolic compounds present in plant extracts are essential to their antimicrobial effects. Our results suggested that the flavonoids exerted a much stronger antimicrobial activity than the polysaccharides probably because of its phenolic compounds. Several studies attribute the inhibitory effect of plant extracts against bacterial pathogens to their phenolic composition. Phenolics not only synergistically act to alter the fluidity of outer and inner membranes (resulting in the release of cell materials to the cytoplasm), but also inhibit energy metabolism, disrupting the electron transport chain and metabolism in microorganisms. In aqueous extracts, the presence of carbohydrates, which may be consumed as a carbon source by microorganisms, may also contribute to the lack of inhibitory effect on the tested microorganisms.

The antioxidant activity of non-volatile plant extracts has been attributed to the presence of phenolic compounds [21]. The antioxidant effect of phenolic compounds is mainly due to their redox properties through various possible mechanisms: free-radical scavenging activity, transition-metal-chelating activity, and/or singlet-oxygen-quenching capacity [22]. In summary, a comparative analysis of the antioxidant activities of the L. aurea bulb extracts proved that the flavonoids exhibited higher antioxidant activity than the polysaccharides. This finding could be related to the total phenolic content because the flavonoids had a higher total phenolic content and better antioxidant activity. Several studies had also pointed out the antioxidant potential of phenolic compounds [23-25].

\section{Conclusion}

The antimicrobial and antioxidant activities of flavonoids and polysaccharides of L. aurea bulbs used in Chinese folk medicine were determined in this study. The total phenolic content in the flavonoids and polysaccharides were also determined. The flavonoids exhibited stronger antimicrobial and antioxidant activities than polysaccharides because the related extract contained a higher total phenolic content. This study was the first to report on the antimicrobial potential of L. aurea bulbs. In light of these valuable bioactivities, the development of natural bacterial inhibitors and antioxidants from $\mathrm{L}$. aurea bulbs is thus important.

\section{Acknowledgments}

This research was funded by Independent Application Project of Agricultural and Social Development Scientific Research in Hangzhou (Grant No. 20191203B31).

\section{References}

[1] R. Al-Tohamy, S. S. Ali, K. Saad-Allah, et al., J. Appl. Biomed. 16(4), 289-300 (2018).

[2] M.-X. Chen, J.-M. Huo, J. Hu, et al., Fitoterapia 130, 48-53 (2018).

[3] Q. Ru, X. Wang, T. Liu, et al., Acta Physiol. Plant. 35(1), 271-282 (2013).

[4] Q. M. Ru, L. R. Zhang, J. D. Chen, et al., Chem. Nat. Compd. 45(4), 474-477 (2009).

[5] Q.-M. Ru, L.-J. Wang, W.-M. Li, et al., Molecules 17(9), 11281-11291 (2012). 
[6] N. Smirnoff and Q. J. Cumbes, Phytochemistry 28(4), 1057-1060 (1989).

[7] A. Braca, N. De Tommasi, L. Di Bari, et al., J. Nat. Prod. 64(7), 892-895 (2001).

[8] H. J. D. Dorman and R. Hiltunen, Food Chem. 88(2), 193-199 (2004).

[9] C. M. Galanakis, E. Tornberg and V. Gekas, J. Chem. Technol. Biot. 85(8), 1148-1155 (2010).

[10] S. Albayrak and A. Aksoy, J. Food Process. Pres. 37(5), 605-614 (2013).

[11] C. Serrano, O. Matos, B. Teixeira, et al., J. Sci. Food Agr. 91(9), 1554-1560 (2011).

[12] P. Y. Chung, L. Y. Chung, Y. F. Ngeow, et al., Pharm. Biol. 42(4-5), 292-300 (2004).

[13] I. Oliveira, A. Sousa, P. Valentão, et al., Food Chem. 105(3), 1018-1025 (2007).

[14] S. A. B. E. Van Acker, D.-j. Van Den Berg, M. N. J. L. Tromp, et al., Free Radical Bio. Med. 20(3), 331-342 (1996).

[15] C. C. Wang, S. C. Chang, B. S. Inbaraj, et al., Food Chem. 120(1), 184-192 (2010).

[16] S. Arabshahi-Delouee and A. Urooj, Food Chem. 102(4), 1233-1240 (2007).

[17] X.-Q. Zha, J.-H. Wang, X.-F. Yang, et al., Carbohyd. Polym. 78(3), 570-575 (2009).

[18] L. Sun, J. Zhang, X. Lu, et al., Food Chem. Toxicol. 49(10), 2689-2696 (2011).

[19] X. Huang, Z. Tu, H. Xiao, et al., Food Bioprod. Process. 91(1), 1-6 (2013).

[20] S. A. Heleno, L. Barros, A. Martins, et al., Food Res. Int. 46(1), 135-140 (2012).

[21] Y. Jiao, P. A. Kilmartin, M. Fan, et al., Food Chem. 268, 77-85 (2018).

[22] B. Shan, Y. Z. Cai, M. Sun, et al., J. Agr. Food Chem. 53(20), 7749-7759 (2005).

[23] D.-Y. Zhang, Y. Wan, J.-Y. Hao, et al., Ind. Crop. Prod. 122, 298-307 (2018).

[24] M. Irakli, P. Chatzopoulou and L. Ekateriniadou, Ind. Crop. Prod. 124, 382-388 (2018).

[25] D. A.Ribeiro, C. J. Camilo, C. de Fátima Alves Nonato, et al., Food Chem., 2020. 315: 1-9. 